\title{
Application of Commercial Non-Dispersive Infrared Spectroscopy Sensors for Sub-ambient Carbon Dioxide Detection
}

\author{
Michael J. Swickrath* and Molly Anderson \\ ${ }^{\dagger}$ NASA Johnson Space Center, Houston, TX, 77058 \\ Summer McMillin \\ ${ }^{\ddagger}$ Engineering and Science Contract Group - Jacobs Technology, Houston, TX, 77058 \\ Craig Broerman \\ ${ }^{\S}$ Engineering and Science Contract Group - Hamilton Sundstrand, Houston, TX, 77058
}

\begin{abstract}
Monitoring carbon dioxide $\left(\mathrm{CO}_{2}\right)$ concentration within a spacecraft or spacesuit is critically important to ensuring the safety of the crew. Carbon dioxide uniquely absorbs light at wavelengths of $3.95 \mu \mathrm{m}$ and $4.26 \mu \mathrm{m}$. As a result, non-dispersive infrared (NDIR) spectroscopy can be employed as a reliable and inexpensive method for the quantification of $\mathrm{CO}_{2}$ within the atmosphere. A multitude of commercial off-the-shelf (COTS) NDIR sensors exist for $\mathrm{CO}_{2}$ quantification. The COTS sensors provide reasonable accuracy so long as the measurements are attained under conditions close to the calibration conditions of the sensor (typically $21.1{ }^{\circ} \mathrm{C}$ and $1 \mathrm{~atm}$ ). However, as pressure deviates from atmospheric to the pressures associated with a spacecraft (8.0-10.2 PSIA) or spacesuit (4.1-8.0 PSIA), the error in the measurement grows increasingly large. In addition to pressure and temperature dependencies, the infrared transmissivity through a volume of gas also depends on the composition of the gas. As the composition is not known a priori, accurate sub-ambient detection must rely on iterative sensor compensation techniques.

This manuscript describes the development of recursive compensation algorithms for sub-ambient detection of $\mathrm{CO}_{2}$ with COTS NDIR sensors. In addition, the basis of the exponential loss in accuracy is developed theoretically considering thermal, Doppler, and Lorentz broadening effects which arise as a result of the temperature, pressure, and composition of the gas mixture under analysis. As a result, this manuscript provides an approach to employing COTS sensors at sub-ambient conditions and may also lend insight into designing future NDIR sensors for aerospace application.
\end{abstract}

*Analyst, Crew and Thermal Systems Division, 2101 NASA Parkway, EC211, Houston, TX, 77058, AIAA Member.

$\dagger$ Analysis Lead, Crew and Thermal Systems Division, 2101 NASA Parkway, EC211, Houston, TX, 77058, AIAA Member.

$\ddagger$ Project Engineer,EVA and Health Systems Group, 2224 Bay Area Blvd., Houston, TX, Member AIAA.

§Project Engineer, CxP and Advanced Systems Group, 2224 Bay Area Blvd., Houston, TX, Member AIAA. 